\title{
Minimum Concentration Normalized by Body Mass Index
}

National Cancer Institute

\section{Source}

National Cancer Institute. Minimum Concentration Normalized by Body Mass Index. NCI

Thesaurus. Code C92374.

The minimum concentration between dose time and dose time plus Tau (at T min) divided by the body mass index. 